\title{
ASYMPTOTIC SOLUTIONS OF HYDRODYNAMIC INTERFACIAL INSTABILITIES IN CYLINDRICAL FLOW
}

\author{
SunG-IK SOHN
}

\begin{abstract}
We present a high-order potential flow model for the motion of hydrodynamic unstable interfaces in cylindrical geometry. The asymptotic solutions of the bubbles in the gravity-induced instability and the shock-induced instability are obtained from the high-order model. We show that the model gives significant high-order corrections for the solution of the bubble.
\end{abstract}

\section{INTRODUCTION}

An interface between two fluids of different densities accelerated by an external force is hydrodynamically unstable. A gravity-driven interfacial instability is known as the Rayleigh-Taylor (RT) instability [1] and a shock-driven interfacial instability is known as the Richtmyer-Meshkov (RM) instability [2]. Both instabilities play important roles in many fields ranging from astrophysics to inertial confinement fusion. These instabilities have been studied in a wide range of contexts $[3,4,5,6$, $7,8,9]$, but many aspects of the dynamics of the instability are still uncertain.

Small perturbations at these unstable interfaces grow into nonlinear structures in the form of bubbles and spikes, and vorticities of mushroom shapes are formed on the spikes [4]. A bubble (spike) is a portion of the light (heavy) fluid penetrating into the heavy (light) fluid. At later times, a bubble in the RT instability attains a constant velocity, while a RM bubble has a decaying growth rate.

One of earliest, and successful, model for RT-type instability is the potential flow model by Layzer [3], applied for the case of infinite density jump. Layzer's model approximates equations locally near the bubble (or spike) tip and gives a set

Received by the editors July 22, 2013. Revised October 13, 2013. Accepted October 22, 2013. 2010 Mathematics Subject Classification. 76B07, 76E17, 76E30.

Key words and phrases. bubble, hydrodynamic instabilities, potential-flow model, asymptotic solution.

This work was supported by the Research Institute of Natural Science of Gangneung-Wonju National University. 
of ordinary differential equations to determine the motion of the bubble (or spike). Since Layzer's work, the model has been studied by many people. Hecht et. al [10] obtained the asymptotic solutions of the bubble of the RM instability of infinite density jump. Goncharov [11] and Sohn [12] then generalized the Layzer-type model to the interfaces of finite density jumps. Sohn [13] succeeded in the modelling of the unstable interfaces with surface tension and viscosity. Recently, Sohn $[14,15]$ extended the model to high-order in two dimensions.

In this paper, we present a high-order Layzer model for the evolution of unstable interfaces in cylindrical geometry and obtain the asymptotic solutions of axisymmetric bubbles in the RT and RM instabilities. Three-dimensional modeling of the interface is an important subject, since the instabilities occur in three dimensions in reality. We will show that the high-order model gives significant corrections in the solutions of the evolution of the three-dimensional unstable interfaces. Note that in two dimensions, there were fairly large differences between the solutions of the low-order model and numerical results, and the high-order model gave improvement on the solutions $[14,15]$.

In Section 2, we describe Layzer's potential-flow model, of low-order, for the evolution of the unstable interface in cylindrical geometry. In Section 3, we present the high-order Layzer model for the motion of the interface. Section 4 gives the time-evolution solutions of the RT and RM bubbles from the high-order model, in comparisons with the low-order model. Section 5 gives conclusions.

\section{Potential-Flow Model}

In this section, we briefly describe the Layzer model for the evolution of unstable interfaces. We consider an interface of infinite density jump (fluid/vacuum) in a cylindrical tube. We assume that the system is axially symmetric (See Fig. 1 in [3]) and the fluid is incompressible, inviscid and irrotational. The evolution of the interface, $z=\eta(r, t)$, is determined by the kinematic condition and the Bernoulli equation

$$
\begin{gathered}
\frac{\partial \eta}{\partial t}+u \frac{\partial \eta}{\partial r}=v \\
\frac{\partial \phi}{\partial t}+\frac{1}{2}\left[\left(\frac{\partial \phi}{\partial r}\right)^{2}+\left(\frac{\partial \phi}{\partial z}\right)^{2}\right]+g \eta=\text { const }
\end{gathered}
$$

where $u$ and $v$ are $r$ and $z$ components of the interface velocity, respectively, and $\mathrm{g}$ 
is an external acceleration. In the RT instability, $g$ is set to a constant value, while the RM instability is modelled by setting $g=0$ and giving a nonzero initial velocity, via the impulsive acceleration [2]. The kinematic condition implies the continuity of the normal component of the fluid velocity across the interface.

The velocity potential of the fluid is taken as

$$
\phi(r, z, t)=a_{1}(t) J_{0}(k r) e^{-k z} .
$$

Here, $k=\beta_{1} / R$ where $\beta_{1} \approx 3.832$ is the first zero of the Bessel function $J_{1}(r)$ and $R$ is the radius of the cylindrical tube. The velocity field of the fluid is defined as

$$
\mathbf{U}=\nabla \phi .
$$

The Stokes stream function corresponding to the velocity potential (2.3) is defined by

$$
\frac{\partial \psi}{\partial z}=-r \frac{\partial \phi}{\partial r}, \quad \frac{\partial \psi}{\partial r}=r \frac{\partial \phi}{\partial z} .
$$

Then, from the relation $d J_{1}(r) / d r=J_{0}(r)-J_{1}(r) / r$, the stream function is

$$
\psi(r, z, t)=-a_{1}(t) r J_{1}(k r) e^{-k z} .
$$

The streamlines generated by $\psi(r, z, t)=\psi\left(r^{*}, z^{*}, t\right)$, passing through an arbitrary reference point $\left(r^{*}, z^{*}\right)$, are given by

$$
z=\frac{1}{k} \ln \left[\frac{r J_{1}(k r)}{r^{*} J_{1}\left(k r^{*}\right)}\right]+z^{*} .
$$

The interface near the tip of the bubble (or the spike) is approximated as

$$
\eta(r, t)=\zeta_{0}(t)+\zeta_{1}(t) r^{2} .
$$

Then, from (2.3) and (2.8), the components of the interface velocity are

$$
u \sim-\frac{1}{2} a_{1} k^{2} e^{-k \zeta_{0}} r, \quad v \sim-a_{1} k e^{-k \zeta_{0}}\left[1-\left(\frac{k^{2}}{4}+k \zeta_{1}\right) r^{2}\right] .
$$

Substituting these expressions into the kinematic condition and equating up to the second order in $r$, one can obtain the following equations

$$
\begin{gathered}
\frac{d \zeta_{0}}{d t}=-a_{1} k e^{-k \zeta_{0}}, \\
\frac{d \zeta_{1}}{d t}=a_{1} k^{2}\left(2 \zeta_{1}+\frac{1}{4} k\right) e^{-k \zeta_{0}} .
\end{gathered}
$$

The second order equation from the Bernoulli equation is given by

$$
k\left(\zeta_{1}+\frac{1}{4} k\right) e^{-k \zeta_{0}} \frac{d a_{1}}{d t}=-a_{1}^{2} k^{3}\left(\zeta_{1}+\frac{1}{8} k\right) e^{-2 k \zeta_{0}}+g \zeta_{1} .
$$


The equations $(2.9) \sim(2.11)$ determine the evolution of the axisymmetric bubble.

The asymptotic solution of the bubble, from the second-order model, can be obtained by taking the large time limit for $(2.9) \sim(2.11)$. The asymptotic velocity and curvature of the RT bubble [3] is

$$
V \rightarrow \sqrt{\frac{g R}{\beta_{1}}}, \quad \zeta_{1} \rightarrow-\frac{\beta_{1}}{8 R},
$$

and the asymptotic velocity and curvature of the RM bubble [10] is

$$
V \sim \frac{R}{\beta_{1} t}, \quad \zeta_{1} \rightarrow-\frac{\beta_{1}}{8 R},
$$

where the bubble velocity represents $V=d \zeta_{0} / d t$. This solution implies that the bubble velocity of the RT bubble converges to a constant limit which is independent on the initial velocity and curvature, and the growth rate of the RM bubble decays to zero. The asymptotic curvatures of the bubbles of the two instabilities are the same.

\section{High-ORDER Model}

We present a high-order extension of the Layzer model. The interface near the tip of the bubble can be written as

$$
z=\eta(r, t)=\sum_{j=0}^{\infty} \zeta_{j}(t) r^{2 j},
$$

and the velocity potential is generalized to

$$
\phi(x, y, t)=\sum_{\substack{j=1 \\ j: \text { odd }}}^{\infty} a_{j}(t) J_{0}\left(k_{j} r\right) e^{-k_{j} z},
$$

where $k_{j}=\beta_{j} / R$ and $\beta_{j}$ is the $j$-th zero of the Bessel function $J_{1}(r)$. Similar to the model in the two dimensions [9], only odd terms are taken in the velocity potential.

The evolution of the interface is again governed by the kinematic condition and the Bernoulli equation. One may apply the similar procedure as Section 2, to derive high-order equations. The velocities at the interface are given approximately by

$$
u=\frac{\partial \phi}{\partial r} \approx-\sum_{j} a_{j} k_{j}^{2}\left[\frac{1}{2} r-k_{j}\left(\frac{1}{2} \zeta_{1}+\frac{1}{16} k_{j}\right) r^{3}\right] e^{-k_{j} \zeta_{0}}
$$




$$
\begin{aligned}
v=\frac{\partial \phi}{\partial y} \approx & -\sum_{j} a_{j} k_{j}\left[1-\left(\frac{1}{4} k_{j}^{2}+k_{j} \zeta_{1}\right) r^{2}\right. \\
& \left.+\left(\frac{1}{2} k_{j}^{2} \zeta_{1}^{2}-k_{j} \zeta_{2}+\frac{1}{4} k_{j}^{3} \zeta_{1}+\frac{1}{64} k_{j}^{4}\right) r^{4}\right] e^{-k_{j} \zeta_{0}}
\end{aligned}
$$

Using this expression and satisfying the kinematic condition up to the fourth order in $r$, we obtain the equations

$$
\begin{gathered}
\frac{d \zeta_{0}}{d t}=-\sum_{j} a_{j} k_{j} e^{-k_{j} \zeta_{0}} \\
\frac{d \zeta_{1}}{d t}=\sum_{j} a_{j} k_{j}^{2}\left(2 \zeta_{1}+\frac{1}{4} k_{j}\right) e^{-k_{j} \zeta_{0}}, \\
\frac{d \zeta_{2}}{d t}=\sum_{j} a_{j} k_{j}^{2}\left(3 \zeta_{2}-\frac{3}{2} k_{j} \zeta_{1}^{2}-\frac{3}{8} k_{j}^{2} \zeta_{1}-\frac{1}{64} k_{j}^{3}\right) e^{-k_{j} \zeta_{0}},
\end{gathered}
$$

where all the summations are taken for $j=1$ and 3 . The second and fourth order equations from the Bernoulli equation are given by

$$
\begin{aligned}
& \sum_{j} k_{j}\left(\zeta_{1}+\frac{1}{4} k_{j}\right) e^{-k_{j} \zeta_{0}} \frac{d a_{j}}{d t}=\frac{1}{8}\left(\sum_{j} a_{j} k_{j}^{2} e^{-k_{j} \zeta_{0}}\right)^{2}-\left(\sum_{j} a_{j} k_{j} e^{-k_{j} \zeta_{0}}\right)\left[\sum_{j} a_{j} k_{j}^{2}\left(\zeta_{1}+\frac{1}{4} k_{j}\right) e^{-k_{j} \zeta_{0}}\right]+g \zeta_{1}, \\
& \sum_{j} k_{j}\left(\zeta_{2}-\frac{1}{2} k_{j} \zeta_{1}^{2}-\frac{1}{4} k_{j}^{2} \zeta_{1}-\frac{1}{64} k_{j}^{3}\right) e^{-j k \zeta_{0}} \frac{d a_{j}}{d t} \\
&=\frac{1}{2}\left[\sum_{j} a_{j} k_{j}^{2}\left(\zeta_{1}+\frac{1}{4} k_{j}\right) e^{-k_{j} \zeta_{0}}\right]^{2}+\left(\sum_{j} a_{j} k_{j} e^{-k_{j} \zeta_{0}}\right) \\
& \times {\left[\sum_{j} a_{j} k_{j}^{2}\left(-\zeta_{2}+\frac{1}{2} k_{j} \zeta_{1}^{2}+\frac{1}{4} k_{j}^{2} \zeta_{1}+\frac{1}{64} k_{j}^{3}\right) e^{-k_{j} \zeta_{0}}\right] } \\
&- \frac{1}{4}\left(\sum_{j} a_{j} k_{j}^{2} e^{-k_{j} \zeta_{0}}\right)\left[\sum_{j} a_{j} k_{j}^{3}\left(\zeta_{1}+\frac{1}{8} k_{j}\right) e^{-k_{j} \zeta_{0}}\right]+g \zeta_{2} .
\end{aligned}
$$

The equations $(3.3) \sim(3.7)$ are the main equations of this paper. The asymptotic solution of the high-order model is not obtained analytically, due to complexity of the equations. We calculate the asymptotic solution by integrating the equations numerically in the next section. 


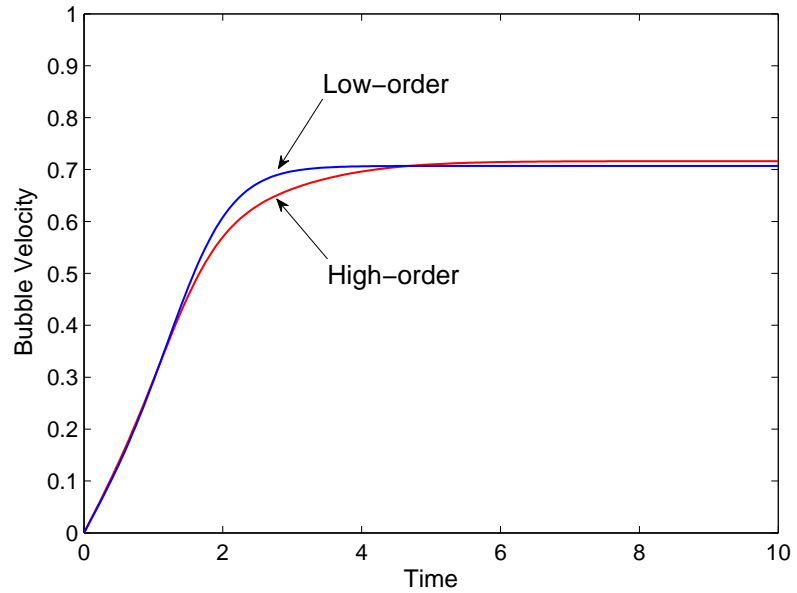

Figure 1. Bubble velocity of the RT instability from the low- and high-order models.

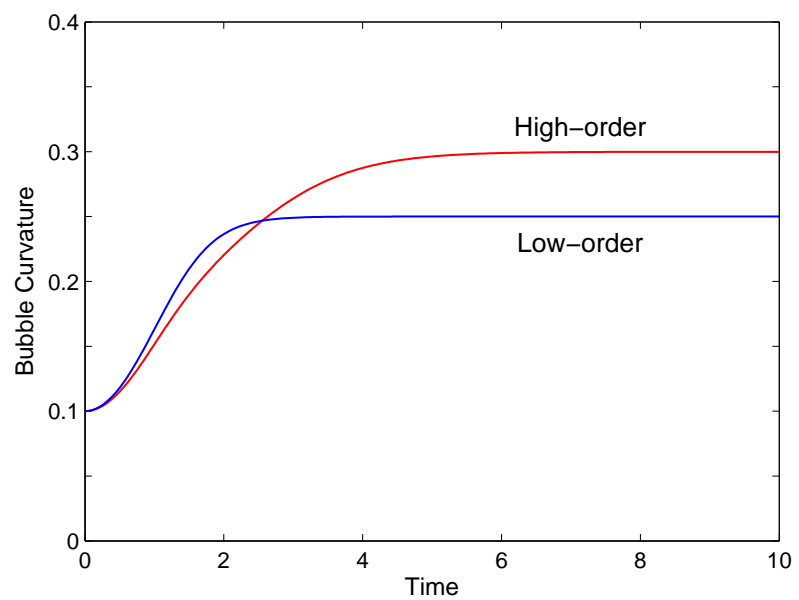

Figure 2. Bubble curvature of the RT instability from the low- and high-order models.

\section{Solutions of the Model}

We now find the solutions of the low- and high-order models, by solving numerically the equations $(2.9) \sim(2.11)$ and (3.3) (3.7), respectively. We employ the standard fourth-order Runge-Kutta method for numerical integrations.

Figure 1 shows the solutions of the bubble velocity of the RT instability from the low- and high-order models. The gravitational acceleration is set to $g=1$ and 


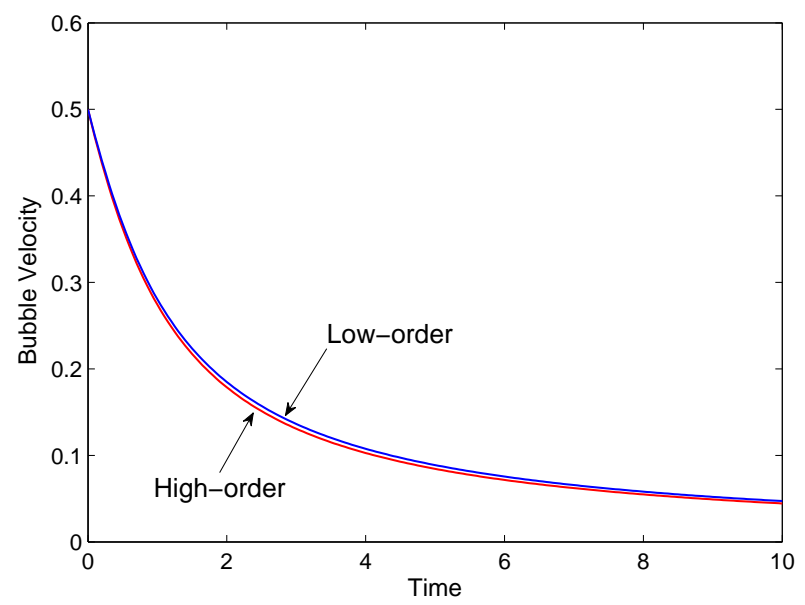

Figure 3. Bubble velocity of the RM instability from the low- and high-order models.

the radius of the tube is $R=\beta_{1} / 2$. The initial amplitude and curvature of the interface are given by $\zeta_{0}=0.1$ and $\zeta_{1}=-0.1$. In Fig. 1 , the bubble velocities from the low-order and high-order models converge to constant limits with small difference. Figure 2 plots the bubble curvature of the RT instability from the lowand high-order models. In Fig. 2, the sign of the curvature is reversed. The highorder solution of the bubble curvature also converges asymptotically to a constant limit. The difference of the asymptotic curvatures of the models is much larger than that of the asymptotic velocity. The terminal values of the bubble curvature of the high-order model is $20 \%$ larger than that of the low-order model.

Figure 3 shows the solutions of the bubble velocity of the RM instability $(g=0)$ from the low- and high-order models. The initial velocity of the interface is given by $V_{0}=0.5$, and the initial amplitude and curvature are the same as the RT case. In Fig. 3, the growth rates of the RM bubble from the low- and high-order models decay to zero at late times. The solution of the high-order model is nearly the same as the low-order solution, but slightly lower. Figure 4 plots the bubble curvature of the RM instability from the models. The solutions of the curvature exhibit a similar feature with the RT instability, but the transient stage at an early time is shorter than the RT instability. The difference of the asymptotic curvatures of the models is again larger than that of the asymptotic velocity. The terminal value of the bubble curvature of the high-order model of the RM instability is smaller than the RT instability. 


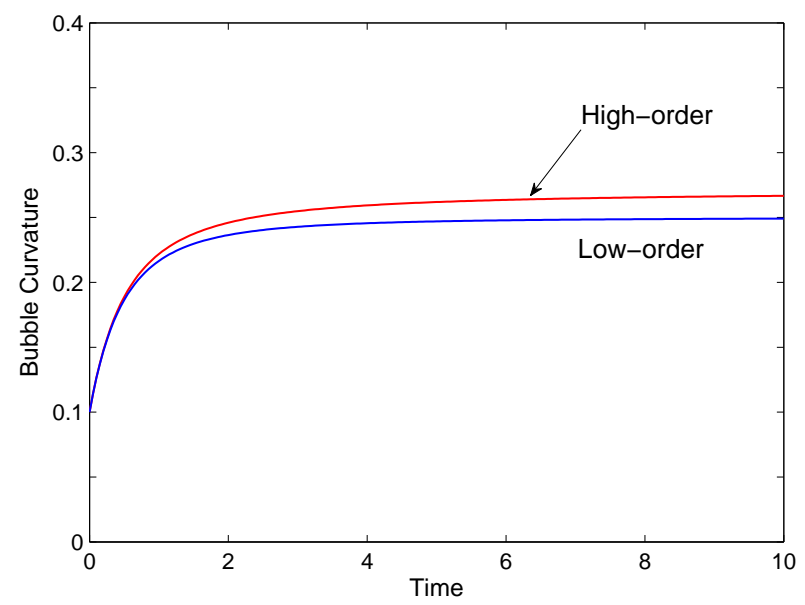

Figure 4. Bubble curvature of the RM instability from the low- and high-order models.

\section{Conclusions}

We have presented the high-order solution for the bubble evolution in the RT and RM instabilities from the Layzer potential-flow model. The model gives significant high-order corrections to the bubble curvature, while the differences of the bubble velocity of the low- and high-order models are small. This feature of the solutions of the Layzer models is similar to the models in two dimensions $[14,15]$.

The present high-order model is developed only for the interface of the infinite density jump. In fact, a high-order Layzer model for the cases of finite density jump could be derived, but we have found that the equations in that model are quite coupled and it is difficult to solve them.

It would be necessary to compare the solutions of the model with results of full numerical simulations, for validation of the model. However, results of numerical simulations for the RT and RM instabilities in cylindrical geometry are very rare. Three-dimensional numerical simulations for the Euler equations are called for.

\section{REFERENCES}

1. Lord Rayleigh: Investigation of the character of the equilibrium of an incompressible heavy fluid of variable density. Proc. London Math. Soc. 14 (1983), 170-177.

2. R.D. Richtmyer: Taylor instability in shock acceleration of compressible fluids. Commun. Pure Appl. Math. 13 (1960), 297-319. 
3. D. Layzer: On the instability of superimposed fluids in a gravitational field. Astrophys. J. 122 (1955), 1-12.

4. D. Sharp: An overview of Rayleigh-Taylor instability. Physica D 12 (1984), 3-10.

5. J. Grove: The interaction of shock waves with fluid interfaces. Adv. Appl. Math. 10 (1989), 201-227.

6. Q. Zhang \& S.-I. Sohn: Quantitative theory of Richtmyer-Meshkov instability in three dimensions. Zeit. Angew. Math. Phys. 50 (1999), 1-46.

7. J. Glimm, J.W. Grove, X.L. Li \& D.C. Tan: Robust computational algorithms for dynamic interface tracking in three dimensions. SIAM J. Sci. Comput. 21 (2000), 20402256.

8. B. Cheng, J. Glimm \& D.H. Sharp: A three-dimensional renormalization group bubble merger model for Rayleigh-Taylor Mixing. Chaos 12 (2002), 267-274

9. S.-I. Sohn: High-resolution vortex simulations of the Richtmyer-Meshkov instability, $J$. Korean Phys. Soc. 60 (2012), 1037-1042.

10. J. Hecht, U. Alon \& D. Shvarts: Potential flow models of Rayleigh-Taylor and Richtmyer-Meshkov bubble fronts. Phys. Fluids 6 (1994), 4019-4030.

11. V.N. Goncharov: Analytic model of nonlinear, single-mode, classical Rayleigh-Taylor instability at arbitrary Atwood numbers. Phys. Rev. Lett. 88 (2002), 134502: 1-4.

12. S.-I. Sohn: Bubble interaction model for hydrodynamic unstable mixing. Phys. Rev. E 75 (2007), 066312: 1-12.

13. _ E E Effects of surface tension and viscosity on the growth rates of Rayleigh-Taylor and Richtmyer-Meshkov instabilities. Phys. Rev. E 80 (2009), 055302: 1-4.

14. __ : A high-order model for spike and bubble in impulsively accelerated interface. Korean J. Math. 20 (2012), 323-331.

15. __ : High-order potential flow model for hydrodynamic unstable interface. $J$. KSIAM 16 (2012), 225-234.

Department of Mathematics, Gangneung-Wonju National University, Gangneung 210702, Republic of Korea

Email address: sohnsi@gwnu.ac.kr 